\title{
IMPACTOS DA NANOTECNOLOGIA NA SAÚDE: PRODUÇÃO DE MEDICAMENTOS
}

\author{
Frantiescoli A. Dimer, Rossana B. Friedrich, Ruy C. R. Beck e Sílvia S. Guterres \\ Faculdade de Farmácia, Universidade Federal do Rio Grande do Sul, 90610-000 Porto Alegre - RS, Brasil \\ Adriana R. Pohlmann* \\ Faculdade de Farmácia, Universidade Federal do Rio Grande do Sul, 90610-000 Porto Alegre - RS, Brasil / Departamento de Química Orgânica, \\ Instituto de Química, Universidade Federal do Rio Grande do Sul, 91501-970 Porto Alegre - RS, Brasil
}

Recebido em 4/7/13; aceito em 14/10/13; publicado na web em 24/10/13

\begin{abstract}
IMPACT OF NANOTECHNOLOGY ON PUBLIC HEALTH: PRODUCTION OF MEDICINES. Several studies have described the benefits of nanoscience and nanotechnology $(\mathrm{N} \& \mathrm{~N})$ in different sectors such as agriculture, energy, environmental preservation, and public health. The rapid evolution of $N \& N$ can be shown through a panoramic analysis of scientific papers and patents. In the area of public health, it is estimated that the global market for nanotechnology products will expand to 160 billion U.S. dollars in 2015. The Brazilian government has also strengthened its innovative potential in N\&N through economic subsidies, as observed for other countries. This review is focused on the current landscape of N\&N in a therapeutic context, highlighting the development of nanotechproducts produced with biocompatible and biodegradable materials that are already commercially available or under investigation. Most studies under investigation are focused on the development of nanotechnology-based formulations intended for treatment of cancer, inflammatory, cardiovascular, and neurological diseases. Although there are several advantages of N\&N in healthcare, many challenges have to be conquered to increase the availability of nanotechnology products in toxicological, preclinical and clinical studies, scale-up, regulatory, and private investments.
\end{abstract}

Keywords: pharmaceutical nanotechnology; nanomedicine; drug.

\section{INTRODUÇÃO}

$\mathrm{O}$ crescente interesse em pesquisas relacionadas à nanociência $\mathrm{e}$ nanotecnologia $(\mathrm{N} \& \mathrm{~N})$ torna esta área um novo patamar do conhecimento, com imensos impactos científicos e econômicos. ${ }^{1}$ Cabe salientar que políticas de Ciência e Tecnologia, C\&T, estão sendo continuamente refletidas e discutidas gerando novas propostas de ações com o objetivo de superar desafios globais apresentados em escala municipal, estadual, regional e/ou nacional. Dentre os temas centrais destas políticas, destacam-se os relacionados à saúde, incluindo o desenvolvimento de fármacos e medicamentos, e matérias-primas e materiais "inovadores e tradicionais", abrangendo a ciência de nanomateriais. ${ }^{2}$

Dados relacionados aos investimentos em N\&N mostram que países como os EUA, Japão e os da Comunidade Europeia lideram financiamentos para o desenvolvimento desse setor, aumentando a competitividade de suas empresas. Estima-se que o mercado mundial de produtos para a saúde de base nanotecnológica crescerá até 160 bilhões de dólares em $2015 .^{3}$ Apesar de financiamentos mais modestos, vários países em desenvolvimento como o Brasil também investem no grande potencial da $\mathrm{N} \& \mathrm{~N}$ e, em decorrência disso, têm apoiado iniciativas nacionais para a promoção dessa área, que poderão se traduzir em melhorias na qualidade de vida de suas populações. Enquanto a nanociência corresponde ao estudo dos fenômenos naturais que regem o comportamento de átomos, moléculas e estruturas na escala nanométrica, a nanotecnologia consiste na aplicação da nanociência para o controle e manipulação da matéria estruturada no nível atômico e molecular, permitindo o desenvolvimento de dispositivos, materiais funcionais e sistemas com propriedades significativamente diferentes daquelas observadas na escala micro- ou macroscópica. ${ }^{4}$ Estudos envolvendo N\&N têm sido direcionados para o desenvolvimento de setores como agricultura, energia, preservação ambiental, saúde pública, entre outros. ${ }^{4}$

\footnotetext{
*e-mail: adriana.pohlmann@ufrgs.br
}

Inúmeros trabalhos científicos descrevem os benefícios da nanotecnologia na terapêutica. A maior parte dos estudos tem sido focada no desenvolvimento de formulações nanotecnológicas destinadas ao tratamento do câncer, de doenças inflamatórias, cardiovasculares, neurológicas e ao combate do vírus da imunodeficiência adquirida (AIDS). Na atualidade, existem mais de uma centena de estudos clínicos com nanomedicamentos em andamento, sendo que a doença-alvo mais recorrente é o câncer de mama, as nanopartículas mais estudadas são as de albumina e o fármaco mais estudado é o paclitaxel. ${ }^{5}$ Contudo a N\&N na área da saúde apresenta inúmeros desafios atuais a serem enfrentados como estudos toxicológicos, pré-clínicos e clínicos, scale-up, regulamentação e de investimentos privados.

Neste contexto, o objetivo desta revisão é analisar o atual panorama da N\&N no âmbito mercadológico e terapêutico, dando destaque aos principais tipos de nanocarreadores utilizados nos produtos nanotecnológicos já comercializados ou em fase de investigação, obtidos a partir de materiais biocompatíveis e biodegradáveis.

\section{TIPOS DE NANOCARREADORES BIODEGRADÁVEIS}

O conceito de nanotecnologia vem sendo aprimorado de acordo com os avanços da ciência, por isso ainda não há um conceito internacional consensual para o termo. Na área da saúde, a European Medicines Agency define a nanotecnologia como o uso de pequenas estruturas menores de 1.000 nanômetros de diâmetro que são projetadas para apresentar propriedades específicas. Particularmente nas áreas de nanotecnologia farmacêutica e de nanomedicina, os esforços em pesquisa e desenvolvimento ( $\mathrm{P} \& \mathrm{D}$ ) visam principalmente projetar nanomedicamentos para oferecerem vantagens em relação aos seus homólogos na forma bulk, principalmente pelo seu tamanho reduzido, ou seja, um aumento na área de superfície em relação ao seu volume. Para tanto, diferentes nanopartículas estão em desenvolvimento, seja para terapêutica ou para diagnóstico, sendo que a tendência 
mais recentemente é o uso combinado dessas funções a partir dos dispositivos "teranósticos". 6

Os materiais utilizados na obtenção de formulações nanotecnológicas são selecionados de acordo com suas características de biodegradabilidade, biocompatibilidade, capacidade para funcionalização de superfície, conjugação, complexação e encapsulamento. ${ }^{7}$ Os fármacos podem ser encapsulados por diferentes mecanismos, que incluem solubilização ou retenção na nanopartícula, conjugação ou adsorção. ${ }^{8}$ Em uma situação ideal as nanopartículas, atuando como nanocarreadores, são capazes de transportar o fármaco para um local de destino específico (receptor, sítio ativo) para exercer a sua atividade terapêutica com o máximo de segurança. Os principais nanocarreadores engenheirados aplicados na terapêutica são: os lipossomas, as nanopartículas lipídicas, as micelas e as nanopartículas poliméricas. ${ }^{7,9}$ Por sua vez, os nanocristais representam uma estratégia de nanomização de fármacos pouco solúveis em água, de forma a aumentar a biodisponibilidade e propiciar vias de administração injetáveis. ${ }^{10}$

\section{Lipossomas}

Lipossomas são estruturas vesiculares formadas por uma ou mais bicamadas concêntricas de lipídeos intermediadas por compartimentos aquosos. Estes sistemas possuem natureza anfifílica, elevada biocompatibilidade e biodegradabilidade, especialmente quando são formulados a partir de lipídeos naturais. Além disso, podem ter seu tamanho, composição lipídica e aquosa, lamelaridade e propriedades de superfície facilmente modificadas de acordo com requisitos farmacêuticos e farmacológicos. ${ }^{11}$ Estas características tornam os lipossomas sistemas altamente versáteis atraindo a atenção dos pesquisadores desde os anos 1970, o que já resultou na introdução de diversos produtos no mercado. Dentre esses, destaca-se o Doxil ${ }^{\circledR}$, que foi um dos primeiros medicamentos com nanotecnologia aprovado nos Estados Unidos pelo FDA em 1995. Este nanomedicamento contém o antitumoral doxorrubicina que é passivamente vetorizado até as células tumorais, após uma diminuição da captação pelo sistema mononuclear fagocitário e aumento no seu tempo de meia-vida na circulação plasmática. ${ }^{12}$ Outros nanomedicamentos utilizando este mesmo fármaco como o DaunoXome ${ }^{\circledR} \mathrm{e} \mathrm{o} \mathrm{Mycet}{ }^{\circledR}$ também já possuem sua aprovação para comercialização. Adicionalmente, o Ambisome ${ }^{\circledR}$ indicado no tratamento de infecções fúngicas, por meio da encapsulação da anfotericina B, é comercialmente disponível desde 1997. Este nanomedicamento possui como vantagem em relação à formulação convencional, a diminuição na incidência de efeitos adversos, possibilitando um aumento da dose administrada. ${ }^{12}$

\section{Nanopartículas lipídicas}

As nanopartículas lipídicas sólidas (NLS) são formadas por uma dispersão de lipídeos com alto ponto de fusão, como, por exemplo, o palmitato de cetila, em meio aquoso. Estudos relatam que uma das vantagens das NLS é o não requerimento de uso de solventes orgânicos na sua produção. Adicionalmente, essas nanopartículas demonstram grande capacidade de encapsular fármacos hidrofílicos e lipofílicos em sua matriz lipídica. ${ }^{13}$ A preparação destes sistemas pode ser realizada por diferentes técnicas, sendo a homogeneização à alta pressão a mais utilizada devido ao seu fácil scale-up e curto tempo de processo. ${ }^{13}$ Apesar de serem utilizadas por diversas vias de administração, as NLS são muito empregadas em cosméticos e medicamentos de uso dermatológico, devido às suas vantagens que incluem: a) excelente biocompatibilidade, pois são compostas por lipídios com baixa toxicidade e citotoxicidade; b) eficiente delivery transdérmico, devido ao pequeno tamanho que garante uma grande área de contato com o estrato córneo, aumentando a quantidade de fármaco que penetra através da pele; e c) alta hidratação da pele, proporcionada devido às propriedades oclusivas deste sistema decorrentes da sua elevada área específica.

No entanto, as formulações de NLS possuem algumas desvantagens, entre elas: o alto teor de água $(70-99 \%)$, que pode levar a vários problemas de estabilidade dos produtos finais que as contém. Como alternativa, em algumas aplicações tópicas, são utilizados os carreadores lipídicos nanoestruturados (CLN). Estes sistemas tem uma menor temperatura de fusão, pois contêm uma adição de óleos (lipídeos líquidos), resultando em um maior teor lipídico (40 - 50\%), que reduz consideravelmente o teor de água na formulação. ${ }^{13}$ Apesar das características das nanopartículas lipídicas, até o momento não existem no mercado medicamentos que as contêm. Por outro lado, esse tipo de nanocarreador é empregado em cosméticos e cosmecêuticos, havendo inúmeros produtos nos mercados internacional e nacional.

\section{Micelas}

Micelas são estruturas anfifílicas amplamente estudadas como sistemas de liberação de fármacos para administração tópica, oral e intravenosa. ${ }^{14} \mathrm{~A}$ literatura relata diversos tipos de sistemas carreadores baseados em micelas, destacando-se os sistemas micelares mistos, as nanopartículas micelares e as micelas poliméricas. Os sistemas micelares mistos são formados a partir de misturas de fosfolipídeos e tensoativos, geralmente ácidos biliares, organizados em torno de um núcleo hidrofóbico contendo a substância lipossolúvel molecularmente dispersa. Esses sistemas possuem tamanhos na faixa de 5 a $60 \mathrm{~nm}$ apresentando boa estabilidade e biocompatibilidade. ${ }^{15}$ No mercado farmacêutico, esta tecnologia está disponível para o fármaco diazepam com o nome comercial de Valium MM $^{\circledR}$ e como suplemento nutricional de vitamina $\mathrm{K}_{1}$ e complexo multivitamínico, os quais possuem os nomes comerciais de Kanakion $\mathrm{MM}^{\circledR}$ e Cerenevit ${ }^{\circledR}$, respectivamente. ${ }^{16}$

As nanopartículas micelares, outro sistema de liberação baseado em micelas, foram desenvolvidas e patenteadas em 2003 pela empresa Novavax, originando o produto comercial Estrasorb ${ }^{\circledR}$ que consiste de uma loção tópica para liberação transdérmica do estradiol. Em termos gerais, esses sistemas constituem-se de uma nanoemulsão multifásica formada após mistura em alta pressão do fármaco, de um solvente, um estabilizante, um óleo e água. O resultado é uma formulação composta por diferentes micro/nanoestruturas que consistem de cristais de fármaco livre, fármaco associado a gotas de óleo, a micelas ou solubilizado na fase contínua. ${ }^{17}$

Em adição, as micelas poliméricas são sistemas autoestruturados anfifílicos que possuem tamanho entre 10 a $200 \mathrm{~nm}$. Caracterizam-se por blocos de copolímeros hidrofílicos e hidrofóbicos organizados ao redor de um núcleo contendo um fármaco lipossolúvel encapsulado, o qual pode interagir de forma não específica ou estar covalentemente ligado aos domínios hidrofóbicos do copolímero. Além disso, macromoléculas hidrofílicas possuindo carga, como ácidos nucleicos, peptídeos e proteínas, também podem ser encapsuladas no núcleo micelar, por meio de interações eletrostáticas com os domínios de carga oposta do polímero, formando os complexos de poliíons. ${ }^{14,18} \mathrm{~A}$ composição do bloco hidrofóbico é altamente variável, o que permite a encapsulação de moléculas com diferentes estruturas, solubilidade e carga iônica, contribuindo para a versatilidade dos sistemas. Por outro lado, o bloco hidrofílico é quase exclusivamente formado por poli(etilenoglicol) (PEG) devido à sua excelente biocompatibilidade $\mathrm{e}$ às propriedades furtivas que minimizam interações indesejáveis com proteínas séricas e componentes celulares. Os polímeros em bloco mais estudados são PEG-poli(aminoácidos), PEG-poli(D,L-lactídeo) (PEG-PLA), PEG-poli(ع-caprolactona) (PEG-PCL) e PEG-poli(óxido 
de propileno)-PEG (PEG-PPO-PEG, Pluronics ${ }^{\circledR}$ ). Apesar dos resultados promissores obtidos em relação às micelas poliméricas, $\mathrm{O}$ mercado ainda não dispõe comercialmente de produtos baseados nesses materiais, porém há vários produtos que se encontram em estudos clínicos de fase I, II e III. ${ }^{18}$

\section{Nanopartículas poliméricas}

Nanopartículas poliméricas representam uma alternativa tecnológica com excelente biocompatibilidade, não imunogênica, não tóxica e biodegradável. ${ }^{8}$ Estes sistemas são transportadores coloidais compostos de polímeros sintéticos ou naturais. Nanopartículas poliméricas são uma classe ampla de nanocarreadores. A mais importante classificação dessas nanopartículas consiste em separá-las em dois grandes grupos: os sistemas vesiculares (nanocápsulas) e os matriciais (nanoesferas). ${ }^{8}$ Diversos polímeros naturais como gelatina, alginato e albumina são utilizados para preparar as nanopartículas, no entanto, eles têm desvantagens, como a difícil reprodutibilidade lote a lote. Por sua vez, os polímeros sintéticos são poliésteres alifáticos, derivados dos ácidos acrílico e metacrílico ou derivados de cianoacrilato de alquila. Os principais exemplos de polímeros pré-formados são: a poli( -caprolactona), o PLA, o poli(glicolídeo) (PLGA) ou PLA-coPLGA. Por outro lado, como exemplos de monômeros para polimerização in situ, destacam-se os derivados de cianoacrilato de alquila. $\mathrm{O}$ fármaco pode encontrar-se dissolvido, retido ou adsorvido na matriz polimérica, ou ainda, no caso de nanocápsulas no núcleo oleoso.

As principais técnicas de preparação das nanopartículas poliméricas são: polimerização in situ, polimerização interfacial, nanoprecipitação, deposição interfacial de polímero, emulsificação-difusão, emulsificação-evaporação de solvente, salting out, e ainda coacervação complexa. ${ }^{19}$ Recentemente, as nanocápsulas de núcleo lipídico, um tipo híbrido de nanopartícula, foram desenvolvidas no Brasil, sendo preparadas por deslocamento de solvente e formadas por auto-organização de seus materiais, lipídicos e poliméricos. ${ }^{20}$

Entre os polímeros naturais, a albumina apresenta várias características únicas que a tornam um ótimo veículo para drug delivery, especialmente na área oncológica. Esta proteína é um transportador natural de moléculas hidrofóbicas, tais como: vitaminas, hormônios e outros constituintes do plasma. A ligação de substâncias hidrofóbicas à albumina é reversível e permite o transporte de fármacos até a superfície das células. Além disso, a albumina facilita a transcitose endotelial dos constituintes acoplados à albumina para o espaço extravascular. Um exemplo de produto no mercado é o Abraxane ${ }^{\circledR}$ (Abraxis BioScience), que contém paclitaxel. Nessa formulação o fármaco encontra-se eficientemente encapsulado em nanopartículas de albumina para a utilização no tratamento do câncer de mama, de células não pequenas do pulmão e de ovário. Anteriormente, já se encontrava disponível o medicamento Taxol ${ }^{\circledR}$ (Bristol-Myers Squibb), que utiliza o Cremophor $\mathrm{EL}^{\circledR}$, como adjuvante na formulação. Entretanto, esse adjuvante pode causar severa alergia, hipersensibilidade e até mesmo choque anafilático. Desta forma, há necessidade da pré-utilização de esteróides e anti-histamínicos para a prevenção destes efeitos adversos. Além disso, outra vantagem do Abraxane ${ }^{\circledR}$ é o seu início de ação mais rápido (30 minutos) em relação ao Taxol (3 horas), aliada à vetorização do fármaco até o tecido tumoral, devido ao carreador. ${ }^{21}$

\section{Nanocristais}

Novos medicamentos têm sido desenvolvidos utilizando nanocristais de fármacos, principalmente por meio da tecnologia NanoCrystal ${ }^{\circledR}$ (Elan Corporation, Ireland). Estudos relatam um aumento na solubilidade de fármacos pouco solúveis e a possibilidade de utilização em formulações pelas vias oral e parenteral. Os nanocristais são produzidos por uma técnica de moagem sob alta energia, utilizando poli(estireno) altamente ramificado, e estabilizados por tensoativo. Esta técnica permite a redução das partículas para um tamanho inferior a $1.000 \mathrm{~nm}$ e, ainda, um aumento na área específica de superfície do fármaco. Por isto, esta tecnologia tem sido utilizada principalmente para um aumento significativo na biodisponibilidade oral de fármacos, reduzindo o tempo para atingir a concentração plasmática máxima. Uma vantagem em relação às nanopartículas poliméricas é a não utilização de matrizes poliméricas. ${ }^{10}$

O comprimido com o imunossupressor sirolimus (Rapamune ${ }^{\circledR}$, Wyeth Pharmaceuticals) foi o primeiro produto comercial disponível no mercado, aprovado em 2000. Alguns outros medicamentos foram re-desenvolvidos empregando a tecnologia NanoCrystal ${ }^{\circledR}$ para uma maior biodisponibilidade e uma menor variação das concentrações plasmáticas do fármaco, quando o paciente está em jejum ou alimentado, como o fenofibrato (Tricor ${ }^{\circledR}$, Abbott), verapamil (Verelan ${ }^{\circledR}$, Alkermes Pharma) e o metilfenidato (Ritalin LA ${ }^{\circledR}$, Novartis).

\section{MEDICAMENTOS NANOTECNOLÓGICOS: DESENVOLVIMENTO E COMERCIALIZAÇÃO}

A evolução de novas tecnologias, inclusive da $N \& N$, pode ser verificada de forma rápida a partir de uma análise do panorama de artigos científicos publicados e, ainda, de patentes depositadas envolvendo-as. Considerando as principais aplicações da nanotecnologia em saúde, um levantamento na Web of Science $^{22}$ revela o câncer como principal alvo dos estudos, seguido pelas aplicações direcionadas ao sistema nervoso central (Figura 1). Os estudos em N\&N crescem exponencialmente na virada do século XXI, sendo que a partir de 2005, se observa um maior destaque para as aplicações relativas ao câncer.

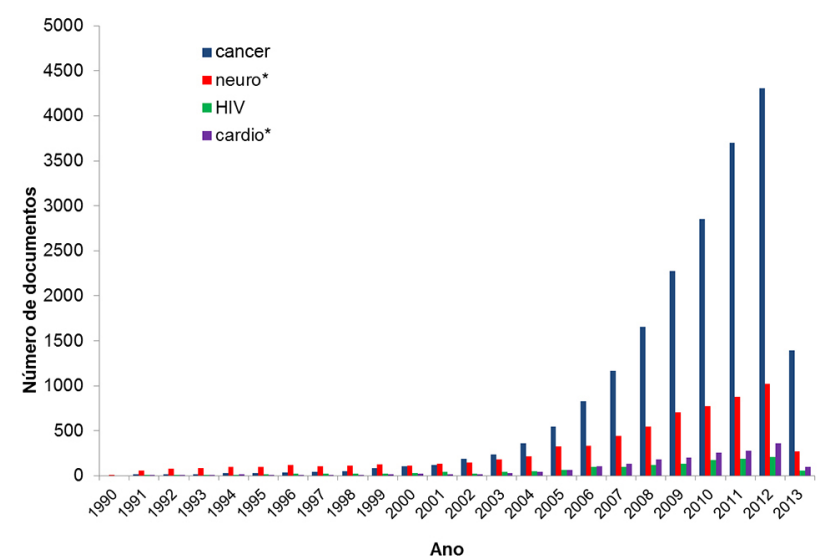

Figura 1. Números de artigos publicados na base de dados Web of Science por ano considerando os cruzamentos: "nano* and cancer", "nano* and neuro*", "nano* and HIV" $e$ "nano* and cardio*”

O depósito de patentes ou de modelos de utilidade representa uma forma de proteção da propriedade intelectual, visando futuras inovações no mercado. Uma das ferramentas utilizadas para levantar o número de patentes depositadas é a base de dados comercial fornecida pela AcclaimiP. ${ }^{23} \mathrm{O}$ levantamento de patentes internacionais, World Intellectual Property Organization (WIPO), evidencia que, embora as primeiras patentes datem do início da década de 1990, para todas as aplicações levantadas, o crescimento exponencial do número de documentos somente é observado a partir da virada do milênio. Cabe salientar que o número de documentos com aplicação em câncer segue crescendo, enquanto que para as demais aplicações levantadas verifica-se um pico em torno de 2008 (Figura 2). 


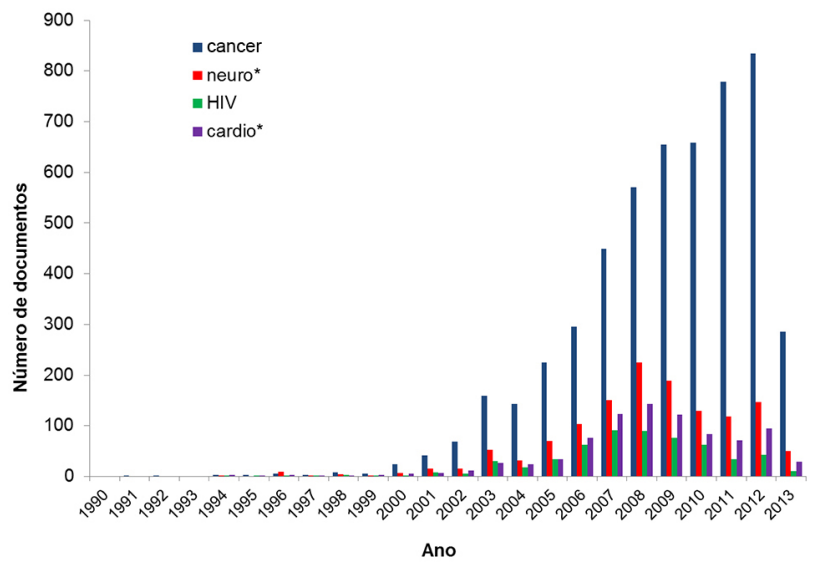

Figura 2. Números de patentes depositadas por ano levantadas na base AcclaimiP considerando os cruzamentos: "nano* and cancer", "nano* and neuro*", "nano* and HIV" e "nano* and cardio*"

A utilização de patentes ou de artigos científicos como métodos indicadores de inovações pode apresentar algumas limitações, pois novos medicamentos nem sempre são detalhados em artigos científicos ou patentes e, ainda, as invenções patenteadas nem sempre irão possuir algum valor econômico ou tecnológico. ${ }^{24}$ Portanto, muitos produtos não são patenteados e muitas patentes não geram produtos. Com isto, mesmo com as inúmeras patentes já depositadas, apenas pouco mais de 20 nanomedicamentos estão disponíveis no mercado mundial (Tabela 1), enquanto que inúmeros outros ainda encontram-se em estudos clínicos. ${ }^{9}$
O maior número de pesquisas direcionadas ao desenvolvimento de produtos nanotecnológicos para o tratamento do câncer já proporcionou, aproximadamente, um rendimento de US\$ 28 bilhões em 2011. Com uma taxa de crescimento anual de $10,8 \%$, a expectativa é de um faturamento próximo a US\$ 50 bilhões em 2016. Por sua vez, os nanomedicamentos destinados às doenças do sistema nervoso central, que faturaram US\$ 14 bilhões em 2011, apresentam um crescimento anual ainda maior, em torno de $16,1 \%$, com um faturamento projetado de US\$ 29,6 bilhões em 2016. ${ }^{25}$

\section{O CENÁRIO DA N\&N VOLTADA À ÁREA DA SAÚDE NO BRASIL}

O investimento do governo brasileiro em N\&N tem crescido de forma expressiva. Em 2012, o Brasil já possuía 24 Redes de Cooperação em nanotecnologia nas diferentes áreas, 16 Institutos de Ciência e Tecnologia que desenvolvem nanotecnologia, 8 Laboratórios Nacionais, mais de 2.500 pesquisadores e 3.000 estudantes (MSc e $\mathrm{PhD}$ ). O Brasil contribui com aproximadamente $1,9 \%$ da produção científica mundial, possuindo a maior e melhor infraestrutura da América Latina. ${ }^{26,27}$ Por meio de um levantamento no Diretório dos Grupos de Pesquisa no Brasil $(\mathrm{CNPq}),{ }^{28}$ foram encontrados 69 grupos de pesquisa brasileiros trabalhando na área da nanotecnologia voltada para a área da saúde, contando com 718 pesquisadores, 999 estudantes e 61 técnicos (Tabela 2). Verifica-se que todas as Regiões do Brasil têm Grupos de Pesquisa atuantes em N\&N na saúde. As Regiões Sudeste (40\%) e Sul (29\%) concentram a maior parte dos Grupos, sendo que $31 \%$ se distribuem nas Regiões Nordeste (16\%), Centro-Oeste (9\%) e Norte (6\%).

Tabela 1. Nanomedicamentos aprovados pelo FDA para comercialização

\begin{tabular}{|c|c|c|c|c|c|c|}
\hline Nome Comercial & Fármaco & Via de administração & Empresa & Indicação & Data de aprovação & Tipo de nanoestrutura \\
\hline Liple & $\begin{array}{l}\text { Palmitato de } \\
\text { Alprostadil }\end{array}$ & i.v. & $\begin{array}{l}\text { Mitsubishi Tanabe } \\
\text { Pharma Corporation }\end{array}$ & $\begin{array}{c}\text { Doença vascular } \\
\text { periférica }\end{array}$ & 1988 & Nanoemulsão lipídica \\
\hline Diprivan & Propofol & i.v. & Zeneca Pharma & Anestésico & 1989 & Lipossomas \\
\hline Adagen & Pegamedase bovina & i.v. & Enzon & $\begin{array}{c}\text { Reposição enzimática } \\
\text { na deficiência de } \\
\text { em pacientes com } \\
\text { imunodeficiência grave }\end{array}$ & 1990 & $\begin{array}{c}\text { Conjugado fármaco- } \\
\text { proteína }\end{array}$ \\
\hline Epaxal & $\begin{array}{l}\text { Vírus inativado da } \\
\text { hepatite A }\end{array}$ & i.m. & Berna Biotech & Vacina contra hepatite A & 1993 & Virossoma \\
\hline Oncaspar & Pegascarpase & s.c. & Enzon & $\begin{array}{l}\text { Leucemia linfoblástica } \\
\text { aguda }\end{array}$ & 1994 & $\begin{array}{l}\text { Conjugado proteína- } \\
\text { polímero } \\
\end{array}$ \\
\hline Neoral & Ciclosporina & Oral & Novartis & Transplante de fígado & 1995 & Microemulsão \\
\hline Doxil/Caelix & Doxorubicina & i.v. & $\begin{array}{l}\text { OrthoBiotech e } \\
\text { Schering-plough }\end{array}$ & $\begin{array}{c}\text { Câncer de ovário com } \\
\text { metastático, Sarcoma de } \\
\text { Kaposi associado a AIDS }\end{array}$ & 1995 & $\begin{array}{c}\text { Lipossomas } \\
\text { peguilados }\end{array}$ \\
\hline Abelcet & Anfotericina B & i.v. & Enzon & Infecções fúngicas & 1995 & $\begin{array}{l}\text { Complexo de } \\
\text { fosfolipídeos }\end{array}$ \\
\hline DaunoXome & Daunorrubicina & i.v. & Gilead Sciences & $\begin{array}{c}\text { Sarcoma de Kaposi } \\
\text { associado a AIDS }\end{array}$ & 1996 & Lipossomas \\
\hline Copaxone & Acetato de Glatirâmer & s.c. & Teva Pharmaceuticals & Esclerose múltipla & 1996 & $\begin{array}{c}\text { Conjugados de } \\
\text { copolímeros }\end{array}$ \\
\hline Amphotec & Anfotericina B & s.c. & Sequus & Infecções fúngicas & 1996 & $\begin{array}{l}\text { Dispersão coloidal de } \\
\text { lipídeos } \\
\end{array}$ \\
\hline Ambisome & Anfotericina B & i.v. & Gilead Sciences & Infecções fúngicas & 1997 & Lipossomas \\
\hline Renagel & Sevelamer & Oral & Genzyme & $\begin{array}{c}\text { Controlar níveis de } \\
\text { fósforo em pacientes com } \\
\text { doença crônica renal em } \\
\text { hemodiálise }\end{array}$ & 1998 & $\begin{array}{l}\text { Nanopartícula } \\
\text { polimérica }\end{array}$ \\
\hline Depocyt & Citarabina & i.v. & SkyePharma e Enzon & Meningite linfomatosa & 1999 & Lipossomas \\
\hline PEGIntron & Peginterferon alfa- $2 b$ & s.c. & $\begin{array}{c}\text { Enzon e Schering- } \\
\text { Plough }\end{array}$ & Hepatite C & 2001 & $\begin{array}{l}\text { Conjugado proteína- } \\
\text { polímero }\end{array}$ \\
\hline
\end{tabular}


Tabela 1. continuação

\begin{tabular}{|c|c|c|c|c|c|c|}
\hline Nome Comercial & Fármaco & Via de administração & Empresa & Indicação & Data de aprovação & Tipo de nanoestrutura \\
\hline Neeulasta & Pegfilgrastim & s.c. & Amgen & Neutropenia & 2002 & $\begin{array}{l}\text { Conjugado proteína- } \\
\text { polímero }\end{array}$ \\
\hline Visudyne & Verteporfin & i.v. & QLT e Novartis & $\begin{array}{c}\text { Degeneração macular } \\
\text { senil }\end{array}$ & 2002 & Lipossomas \\
\hline Ritalin LA & Metilfenidato & Oral & Novartis & $\begin{array}{c}\text { Estimulante do Sistema } \\
\text { Nervoso Central }\end{array}$ & 2002 & Nanocristal \\
\hline Pegasys & $\begin{array}{l}\text { Peginterferon alfa-2a } \\
\text { recombinante }\end{array}$ & s.c. & $\begin{array}{c}\text { Nektar e Hoffmann- } \\
\text { La Roche }\end{array}$ & Hepatite B e C & 2002 & $\begin{array}{c}\text { Conjugado proteína- } \\
\text { polímero }\end{array}$ \\
\hline Somavert & Pegvisomanto & s.c. & Nektar e Pfizer & Acromegalia & 2003 & $\begin{array}{c}\text { Conjugado fármaco- } \\
\text { proteína }\end{array}$ \\
\hline Estrasorb & $\begin{array}{l}\text { Hemihidrato de } \\
\text { estradiol }\end{array}$ & Transdérmica & Novavax & $\begin{array}{c}\text { Tratamento da } \\
\text { menopausa }\end{array}$ & 2003 & Micelas \\
\hline Macugen & Pegaptanibe sódico & Intravitreal & $\begin{array}{c}\text { OSI Pharmaceutics e } \\
\text { Pfizer } \\
\end{array}$ & Degeneração macular & 2004 & $\begin{array}{l}\text { Oligonucleotídeo } \\
\text { peguilado }\end{array}$ \\
\hline Abraxane/Taxol & Paclitaxel & i.v. & $\begin{array}{c}\text { Abraxis, BioScience e } \\
\text { Astrazeneca }\end{array}$ & Câncer de mama & 2005 & $\begin{array}{c}\text { Conjugado fármaco- } \\
\text { albumina }\end{array}$ \\
\hline Triglide & Fenofibrato & Oral & $\begin{array}{c}\text { SkyePharma e First } \\
\text { Horizon }\end{array}$ & $\begin{array}{c}\text { Desordens lipídicas } \\
\text { (colesterol e } \\
\text { triglicerideos) } \\
\end{array}$ & 2005 & Nanocristal \\
\hline Elestrin & Estradiol & Transdérmica & BioSanté & Sintomas da menopausa & 2006 & $\begin{array}{l}\text { Nanopartícula de } \\
\text { fosfato }\end{array}$ \\
\hline Cimzia & Certolizumabe pegol & i.m. & Enzon & $\begin{array}{c}\text { Artrite reumatóide e } \\
\text { doença de Crohn }\end{array}$ & 2008 & $\begin{array}{c}\text { Nanopartícula } \\
\text { polimérica }\end{array}$ \\
\hline Durezol & Difluprednate & Tópico & Sirion Therapeutics & $\begin{array}{c}\text { Anti-inflamatório } \\
\text { oftálmico }\end{array}$ & 2008 & Microemulsão \\
\hline Ferumoxytol & Suplemento de Ferro & i.v. & $\begin{array}{c}\text { Amag } \\
\text { Pharmaceuticals } \\
\end{array}$ & Anemia & 2009 & $\begin{array}{c}\text { Nanopartículas } \\
\text { magnéticas }\end{array}$ \\
\hline
\end{tabular}

Tabela 2. Grupos de Pesquisa Diretório CNPq voltados à nanotecnologia para a terapêutica no Brasil

\begin{tabular}{|c|c|c|c|c|c|}
\hline Grupo de Pesquisa & Instituição & Estado & Pesquisadores & Estudantes & Técnicos \\
\hline Avaliação de Fármacos, Medicamentos e Serviços Farmacêuticos & UEPG & $\mathrm{PR}$ & 6 & 18 & 1 \\
\hline Avaliação Farmacológica e Modelagem PK/PD & UFRGS & RS & 6 & 23 & 1 \\
\hline Biomateriais e Nanotecnologia & UNESP & SP & 5 & 10 & 0 \\
\hline Biomateriais para Odontologia & UFF & RJ & 4 & 6 & 1 \\
\hline $\begin{array}{l}\text { Biomaterias: Preparação, Caracterização, Modelagem Teórica e Aplicações } \\
\text { Biomédicas }\end{array}$ & $\mathrm{CBPF}$ & RJ & 14 & 4 & 2 \\
\hline Biotecnologia Ambiental e Saúde Pública & UTFPR & PR & 11 & 11 & 2 \\
\hline Biotecnologia aplicada à Saúde & UNP & $\mathrm{RN}$ & 9 & 9 & 0 \\
\hline Biotecnologia e Inovação Terapêutica & UFS & SE & 30 & 47 & 0 \\
\hline Biotecnologia Farmacêutica & UNICAMP & SP & 3 & 5 & 0 \\
\hline Caracterização e Desenvolvimento de Biomateriais & UFPEL & RS & 25 & 79 & 1 \\
\hline Células-tronco e Nanotecnologia para a Engenharia de Tecidos & UFRGS & RS & 16 & 19 & 0 \\
\hline $\begin{array}{l}\text { Correlação entre a estrutura e as propriedades de materiais com aplicação em } \\
\text { farmácia }\end{array}$ & UNESP & SP & 2 & 5 & 0 \\
\hline Desenvolvimento de Medicamentos & UFRN & $\mathrm{RN}$ & 25 & 61 & 0 \\
\hline Desenvolvimento de Novas Formulações Farmacêuticas. & UFRJ & $\mathrm{RJ}$ & 5 & 5 & 2 \\
\hline Desenvolvimento de sistemas multiparticulados & UFSC & SC & 5 & 14 & 2 \\
\hline $\begin{array}{l}\text { Desenvolvimento de sistemas nanoparticulados contendo fitoterápicos com } \\
\text { atividade biológica }\end{array}$ & UFRJ & RJ & 2 & 2 & 0 \\
\hline Desenvolvimento e Análise de Produtos para a Saúde & UNIPAR & PR & 15 & 23 & 4 \\
\hline Desenvolvimento e Avaliação Biológica de Medicamentos & UFOP & MG & 21 & 11 & 0 \\
\hline Desenvolvimento e avaliação de formas farmacêuticas e cosméticas & USP & SP & 5 & 3 & 1 \\
\hline Desenvolvimento galênico e nanotecnologia de produtos naturais & UFF & $\mathrm{RJ}$ & 6 & 10 & 0 \\
\hline Desenvolvimento Tecnológico de Medicamentos/Cosméticos e Métodos Analíticos & UFMS & MS & 6 & 9 & 0 \\
\hline $\begin{array}{l}\text { Desenvolvimento Tecnológico e Controle da Qualidade de Fármacos e Medi- } \\
\text { camentos }\end{array}$ & Unicentro & PR & 2 & 5 & 0 \\
\hline Doenças Infecciosas e Resistência Microbiana & UFPE & $\mathrm{PE}$ & 12 & 15 & 2 \\
\hline $\begin{array}{l}\text { DPLF - Núcleo de Desenvolvimento de Dispositivos Poliméricos de Liberação } \\
\text { de Fármacos }\end{array}$ & UFMG & MG & 18 & 10 & 0 \\
\hline
\end{tabular}


Tabela 2. continuação

\begin{tabular}{|c|c|c|c|c|c|}
\hline Grupo de Pesquisa & Instituição & Estado & Pesquisadores & Estudantes & Técnicos \\
\hline Farmacotecnica & UFPR & PR & 12 & 45 & 0 \\
\hline Fotoquímica e Saúde & UNB & DF & 2 & 13 & 1 \\
\hline $\begin{array}{l}\text { Grupo de Desenvolvimento de Materiais estratégicos para processamento de Bio- } \\
\text { massa em Biorefinarias e de Nanomateriais aplicados a Nanomedicina e Virologia }\end{array}$ & UNESP & SP & 13 & 9 & 0 \\
\hline Grupo de Pesquisa em Ciências Farmacêuticas e Saúde Pública & UFAM & $\mathrm{AM}$ & 10 & 23 & 0 \\
\hline Grupo de Pesquisa em Cirurgia Experimental & UFRN & $\mathrm{RN}$ & 5 & 4 & 2 \\
\hline Grupo de Pesquisa em Fármacos & UNIFAP & PA & 26 & 13 & 1 \\
\hline Grupo de Pesquisa em Nanobiotecnologia e Nanotoxicologia & UNIPAMPA & RS & 6 & 9 & 0 \\
\hline Grupo de Pesquisa em Nanoestruturas e Interfaces Biológicas & UFPE & PE & 7 & 4 & 1 \\
\hline Grupo de Pesquisas em Nanotecnologia e Nanomateriais & CEFET & MG & 5 & 9 & 1 \\
\hline Inflamação Intestinal & USP & SP & 3 & 5 & 4 \\
\hline Laboratório de Compostos Bioativos & UFABC & SP & 3 & 13 & 0 \\
\hline Laboratório de Micro e Nanopartículas Aplicadas na Terapêutica & UFRGS & RS & 15 & 18 & 0 \\
\hline Medicamentos Oftálmicos & UNIFESP & SP & 15 & 8 & 5 \\
\hline Micro e Nanotecnologia Farmacêutica & UNESP & SP & 5 & 8 & 4 \\
\hline NanoBioMAv & UFABC & SP & 12 & 8 & 0 \\
\hline Nanobiotecnologia Aplicada à Saúde & UFMG & MG & 17 & 19 & 0 \\
\hline Nanobiotecnologia e Saúde & UNB & $\mathrm{DF}$ & 8 & 14 & 0 \\
\hline Nanociência, Nanotecnologia e Nanobiotecnologia (NNNb) & UFAC & $\mathrm{AC}$ & 13 & 17 & 0 \\
\hline Nanomaterias para aplicações biomédicas & UNIFESP & SP & 2 & 4 & 0 \\
\hline Nanorradiofármacos e Novos Radiofármacos & UFRJ & RJ & 41 & 24 & 0 \\
\hline Nanotecnologia & UEM & PR & 2 & 1 & 0 \\
\hline Nanotecnologia, Biotecnologia e Cultura de Células - NBC & UFPE & $\mathrm{PE}$ & 6 & 4 & 0 \\
\hline Nanotecnologia e Sistemas de Liberação de Fármacos & IFRJ & RJ & 5 & 7 & 0 \\
\hline Núcleo de Estudos em Cariologia e Odontopediatria - NECO & FURB & $\mathrm{SC}$ & 4 & 1 & 0 \\
\hline Núcleo de Pesquisa e Inovação em Ciências da Saúde & UFJF & MG & 5 & 7 & 1 \\
\hline Núcleo de Pesquisa em Nanociências & UNICENTRO & $\mathrm{PR}$ & 3 & 11 & 1 \\
\hline Pesquisa e Desenvolvimento de Sistemas de Liberação de Fármacos & FUNED & MG & 16 & 2 & 0 \\
\hline $\begin{array}{l}\text { Pesquisa, Desenvolvimento, Produção e Controle de Qualidade de Fármacos, } \\
\text { Medicamentos e Cosméticos }\end{array}$ & UFMT & MT & 6 & 3 & 0 \\
\hline Pesquisa e Desenvolvimento de Sistemas de Liberação Transdérmica de Drogas & USP & SP & 24 & 6 & 2 \\
\hline Pesquisa e Desenvolvimento de sistemas para liberação modificada de fármacos & UEM & PR & 7 & 2 & 0 \\
\hline $\begin{array}{l}\text { Pesquisa e Desenvolvimento de Sistemas de Liberação de Fármacos Baseados } \\
\text { em Nanotecnologia }\end{array}$ & UNESP & SP & 4 & 6 & 2 \\
\hline Pesquisa in Pharma & UEPG & PR & 10 & 6 & 2 \\
\hline $\begin{array}{l}\text { Planejamento e Desenvolvimento de Fármacos em Doenças Tropicais, Estresse } \\
\text { Oxidativo e HIV. }\end{array}$ & UFPA & PA & 9 & 18 & 1 \\
\hline Produtos Naturais Aplicados à Saúde & UNIFOR & $\mathrm{CE}$ & 25 & 12 & 0 \\
\hline QUITECH & UFSC & $\mathrm{SC}$ & 4 & 5 & 0 \\
\hline Sistemas de liberação controlada de fármacos e de outras substâncias ativas & UNESP & SP & 7 & 5 & 0 \\
\hline $\begin{array}{l}\text { Sistemas de liberação controlada de fármacos e vacinas: Nanotecnologia } \\
\text { Farmacêutica }\end{array}$ & UFPE & $\mathrm{PE}$ & 25 & 26 & 1 \\
\hline Sistemas de Liberação de Fármacos e Biofarmácia & UEPB & $\mathrm{PB}$ & 9 & 10 & 1 \\
\hline $\begin{array}{l}\text { Sistemas de liberação de fármacos para tratamento de doenças tropicais e emer- } \\
\text { gentes }\end{array}$ & UNESP & SP & 9 & 10 & 0 \\
\hline Sistemas micro- e nanoparticulados contendo substâncias de interesse farmacêutico & UFSM & RS & 7 & 34 & 0 \\
\hline Sistemas nanoestruturados para administração de fármacos & UFRGS & RS & 11 & 25 & 0 \\
\hline Tecnologia dos Alimentos e Farmacêutica & UNB & $\mathrm{DF}$ & 18 & 28 & 5 \\
\hline Tecnologia e Controle de Qualidade de Medicamentos e Alimentos & UFAL & $\mathrm{AL}$ & 4 & 61 & 3 \\
\hline Tecnologia e Nanotecnologia Farmacêutica & UFG & GO & 9 & 18 & 3 \\
\hline Toxicologia Analítica e Metabólica - LATOX & UFRGS & RS & 16 & 20 & 1 \\
\hline TOTAL: 69 grupos de pesquisa & & & 718 & 999 & 61 \\
\hline
\end{tabular}

O Governo brasileiro tem fortalecido o seu potencial inovador em $\mathrm{N} \& \mathrm{~N}$ principalmente por meio de editais do CNPq e da Financiadora de Estudos e Projetos (FINEP), voltados à inovação de produtos ou processos tecnológicos, inclusive para o desenvolvimento da nanotecnologia. Entre 2000 e 2007, o Governo investiu por meio de suas universidades e centros de pesquisa, R \$ 160 milhões em nanotecnologia. ${ }^{4}$ Somando os investimentos do setor privado, estima-se um total de R\$ 320 milhões neste período. Os recursos, ainda que 
modestos quando comparados ao investimento de outros países, como os Estados Unidos, que investiram mais de $\$ 15$ bilhões nos últimos 10 anos em nanotecnologia, ${ }^{29}$ permitiram que diversas redes de pesquisa em nanotecnologia no Brasil se consolidassem.

Entre os recursos alocados em subvenção econômica para a inovação em nanotecnologia em empresas privadas destacam-se os investimentos recebidos pelas empresas Biolab Sanus Farmacêutica Ltda., Biosintética Farmacêutica (Aché Laboratórios Farmacêuticos), Natura Cosméticos, Biocancer - Centro de Pesquisa e Tratamento de Câncer Ltda, Chemyuniom Química Ltda., EMS S.A., Cristália Produtos Químicos, Chron Epigen Indústria e Comércio Ltda. Em um futuro próximo, novos investimentos ainda serão recebidos por estas e outras empresas por meio do edital publicado em Agosto de 2013 (FINEP/MCTI/MS/CNPq), que conta com apoio à inovação tecnológica no setor de saúde - Inova Sáude. Com estes investimentos, existe a tendência de uma maior agilidade da implementação de novos produtos farmacêuticos relacionados à N\&N.

\section{DESAFIOS, GARGALOS E TENDÊNCIAS}

Embora os investimentos em N\&N no Brasil sejam modestos quando comparados aos países desenvolvidos, observa-se claramente a formação de uma massa crítica de pesquisadores bastante produtivos, distribuídos em várias universidades brasileiras. Os resultados dos ensaios pré-clínicos brasileiros mostram uma capacidade inventiva, sendo que produtos de base nanotecnológica de aplicação dermatológica destinados à terapêutica já se encontram em fase I. $\mathrm{O}$ crescimento das interações universidade-empresa envolvendo transferência de tecnologia poderá resultar no aumento da inovação em produtos nanotecnológicos. A cultura de proteção da produção intelectual é uma das chaves para o aumento dessas interações. Por outro lado, os principais desafios nacionais para o crescimento da inovação estão relacionados com os gargalos de: a) criar infraestrutura para a produção de lotes-piloto para ensaios clínicos em fase I; b) incrementar os investimentos para P,D\&I e N\&N; e c) estabelecer marco regulatório brasileiro para $\mathrm{N} \& \mathrm{~N}$ na perspectiva internacional.

\section{CONCLUSÕES}

Os medicamentos nanotecnológicos oferecem comodidade e maior eficiência terapêutica para os pacientes. Além disso, para as empresas, são capazes de estender a rentabilidade econômica dos medicamentos patenteados e criar uma nova fonte de receita. Atualmente, diversos produtos contendo nanopartículas são objetos de estudos pré-clínicos e clínicos nos países desenvolvidos e em desenvolvimento, confirmando a grande promessa dos últimos anos de pesquisa.

\section{AGRADECIMENTOS}

Os autores agradecem o financiamento de pesquisa das Agências Brasileiras: CNPq/MCTI, FAPERGS, PRONEX e PRONEM FAPERGS/CNPq e CAPES/MEC.

\section{REFERÊNCIAS}

1. Pohlmann, A. R.; Beck, R. C.; J. Biomed. Nanotechnol. 2012, 8, 191.

2. Pinto, A. C.; Zucco, C.; Galembeck, F.; de Andrade, J. B.; Vieira, P. C.; Quim. Nova. 2012, 35, 2092.

3. Shi, J.; Votruba, A. R.; Farokhzad, O. C.; Langer, R.; Nano Lett. 2010 , 10,3223

4. ABDI; Panorama nanotecnologia, disponível em http://www.abdi.com. br/Estudo/Panorama\%20de\%20Nanotecnologia.pdf, acessada em Junho de 2013.

5. http://www.clinicaltrial.gov, acessada em Outubro de 2012.

6. Lammers, T.; Aime, S.; Hennink, W. E.; Storm, G.; Kiessling, F.; Acc. Chem. Res. 2011, 44, 1029.

7. Moghimi, S. M.; Hunter, A. C.; Murray, J. C.; The FASEB Journal 2005, $19,311$.

8. Schaffazick, S. R.; Guterres, S. S.; Freitas, L. de L.; Pohlmann, A. R.; Quim. Nova 2003, 26, 726 .

9. Etheridge, M. L.; Campbell, S. A.; Erdman, A. G.; Haynes, C. L.; Wolf, S. M.; McCullough, J.; Nanomed. Nanotechnol. Biol. Med. 2013, 9, 1.

10. Durán, N.; Durán, M.; Tasic, L.; Marcato, P. D.; Quim. Nova 2010, 33, 151.

11. Santos, N. C.; Castanho, M. A. R. B.; Quim. Nova 2002, 25, 1181.

12. Barenholz, Y.; J. Controlled Release 2012, 160, 117.

13. Pardeike, J.; Hommoss, A.; Müller, R. H.; Int. J. Pharm. 2009, 366, 170.

14. Torchilin, V. P.; Pharm. Res. 2007, 24, 1.

15. Almgren, M.; Biochim. Biophys. Acta, Biomembr. 2000, 1508, 146.

16. Rupp, C.; Steckel, H.; Müller, B. W.; Int. J. Pharm. 2010, 395, 272.

17. Lee, R. W.; Shenoy, D. B.; Sheel, R.; Handbook of Non-Invasive Drug Delivery Systems; Vitthal, S. K., ed.; William Andrew Publishing: Boston, 2010, cap. 2.

18. Miyata, K.; Christie, R. J.; Kataoka, K.; React. Funct. Polym. 2011, 71, 227.

19. Mora-Huertas, C. E.;Fessi, H.;Elaissari, A.; Int. J. Pharm. 2010, 385, 113.

20. Jornada, D. S.; Fiel, L. A.; Bueno, K.; Gerent, J. F.; Petzhold, C. L.; Beck, R. C. R.; Guterres, S. S.; Pohlmann, A. R.; Soft Matter 2012, 8 , 6646.

21. Hawkins, M. J.; Soon-Shiong, P.; Desai, N.; Adv. Drug. Deliv. Rev. 2008, 60,876 .

22. http://apps.webofknowledge.com, acessada em Junho de 2013.

23. https://www.acclaimip.com/, acessada em Junho de 2013.

24. Sant'Anna, L. de S.; Alencar, M. S. de M.; Ferreira, A. P.; Quim. Nova 2013, 36, 348 .

25. BCC_Research; Nanotechnology in Medical Applications: The Global Market, disponível em http://www.etp-nanomedicine.eu/public/newsevents/news-archive-1/new-market-research-report-nanotechnology-inmedical-applications-the-global-market, acessada em Junho de 2013.

26. Silva, F. B.; Iniciativa Brasileira de Nanotecnologia disponível em http://www.desenvolvimento.gov.br/arquivos/dwnl_1336677327.pdf, acessada em Junho de 2013.

27. Pohlmann, A. R.; Beck, R. C. R.; J. Biomed. Nanotechnol. 2012, 8, 191.

28. http://dgp.cnpq.br/buscaoperacional/, acessada em Junho de 2013.

29. Sargent, J. F.; The National Nanotechnology Initiative: Overview, Reauthorization, and Appropriations Issues disponível em http://www. fas.org/sgp/crs/misc/RL34401.pdf, acessada em Junho de 2013. 